\title{
Intravital imaging reveals p53-dependent cancer cell death induced by phototherapy via calcium signaling
}

\author{
Carlotta Giorgi ${ }^{1, *}$, Massimo Bonora ${ }^{1, *}$, Sonia Missiroli ${ }^{1}$, Federica Poletti ${ }^{1}$, Fabian \\ Galindo Ramirez ${ }^{2,3}$, Giampaolo Morciano ${ }^{1}$, Claudia Morganti ${ }^{1}$, Pier Paolo Pandolfi ${ }^{4}$, \\ Fabio Mammano ${ }^{2}$ and Paolo Pinton ${ }^{1}$ \\ ${ }^{1}$ Department of Morphology, Surgery and Experimental Medicine, Section of Pathology, Oncology and Experimental Biology \\ and LTTA center, University of Ferrara, Ferrara, Italy \\ 2 Department of Physics and Astronomy, University of Padua, and Venetian Institute of Molecular Medicine, Padua, Italy \\ ${ }^{3}$ Instituto de fisiologia, Benemerita Universidad Autónoma de Puebla (BUAP), Puebla, Mexico \\ ${ }^{4}$ Cancer Genetics Program, Beth Israel Deaconess Cancer Center, Departments of Medicine and Pathology, Beth Israel \\ Deaconess Medical Center, Harvard Medical School, Boston, MA, USA \\ * These authors contributed equally to this work \\ Correspondence to: Paolo Pinton, email: pnp@unife.it \\ Keywords: Calcium $\left(\mathrm{Ca}^{2+}\right)$, cell death, apoptosis, TRP53 (p53), mitochondria
}

Received: July 20, 2014

Accepted: December 01, 2014

Published: December 02, 2014

This is an open-access article distributed under the terms of the Creative Commons Attribution License, which permits unrestricted use, distribution, and reproduction in any medium, provided the original author and source are credited.

\section{ABSTRACT}

One challenge in biology is signal transduction monitoring in a physiological context. Intravital imaging techniques are revolutionizing our understanding of tumor and host cell behaviors in the tumor environment. However, these deep tissue imaging techniques have not yet been adopted to investigate the second messenger calcium $\left(\mathrm{Ca}^{2+}\right)$. In the present study, we established conditions that allow the in vivo detection of $\mathrm{Ca}^{2+}$ signaling in three-dimensional tumor masses in mouse models. By combining intravital imaging and a skinfold chamber technique, we determined the ability of photodynamic cancer therapy to induce an increase in intracellular $\mathrm{Ca}^{2+}$ concentrations and, consequently, an increase in cell death in a p53-dependent pathway.

\section{INTRODUCTION}

The development of malignant tumors results from the deregulated proliferation of cells or from the inability of cells to undergo apoptotic death [1]. Consequently, apoptosis induction in tumor cells is one of the aims of anti-cancer therapy. Studies during the past decade have highlighted the importance of calcium $\left(\mathrm{Ca}^{2+}\right)$ signaling in the regulation of key aspects of cell death. In particular, the modulation of $\mathrm{Ca}^{2+}$ signaling can change cell sensitivity to apoptotic signals, such as chemotherapeutic agents [2].

Several studies have indicated that the $\mathrm{Ca}^{2+}$ content of the endoplasmic reticulum (ER) determines cell sensitivity to apoptotic stress and that perturbation of ER $\mathrm{Ca}^{2+}$ homeostasis appears to be a key component in the development of several pathologies. Therefore, changes in the $\mathrm{Ca}^{2+}$ flux are regulated by $\mathrm{Ca}^{2+}$ release from the ER store toward the cytosol, and mitochondria can promote the survival of cancer cells, reducing sensitivity to apoptotic signals [3]. Although the majority of the mechanisms related to intracellular $\mathrm{Ca}^{2+}$ transport have been successfully elucidated either in vitro or in cultured cells, we still know little regarding the actual physiological role of these processes in the context of the tumor environment primarily due to technical limitations. However, recent advancements in imaging have allowed researchers to visualize transient changes in $\mathrm{Ca}^{2+}$ levels in live mice [4].

We recently demonstrated that tumor suppressors could modulate cell sensitivity to apoptosis by regulating $\mathrm{Ca}^{2+}$ transfer from the endoplasmic reticulum (ER) to mitochondria [5-7]. However, this intriguing signaling pathway has never been investigated in the tumor environment.

This study is the first to demonstrate the direct measurements of intracellular $\mathrm{Ca}^{2+}$ dynamics in vivo within tumor masses. Importantly, the methodology used in the present study is readily applicable to all the currently available fluorescent probes for following any intracellular parameter of interest. Finally, in the present 
study, we demonstrate $\mathrm{Ca}^{2+}$-dependent p53-mediated apoptosis induced by phototherapy in cancer cells.

This approach will generate new opportunities for elucidating chemoresistance signaling in tumors and for developing new anti-cancer therapy.

\section{RESULTS}

We adopted the well-known tumor suppressor p53 to generate a tumor model related to the loss of an onco-suppressor $[8,9]$. The mechanism of action of p53 is dependent on its transcription factor activity. Recently, a cytoplasmic fraction of p53 was suggested to directly modulate apoptosis via its cytoplasmic localization [10],

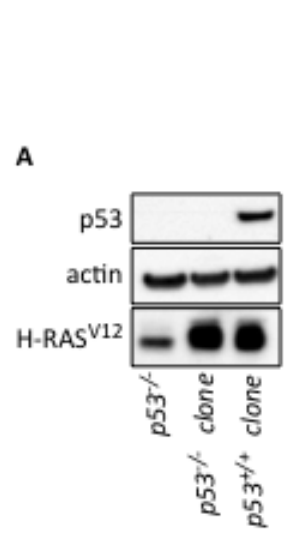

B
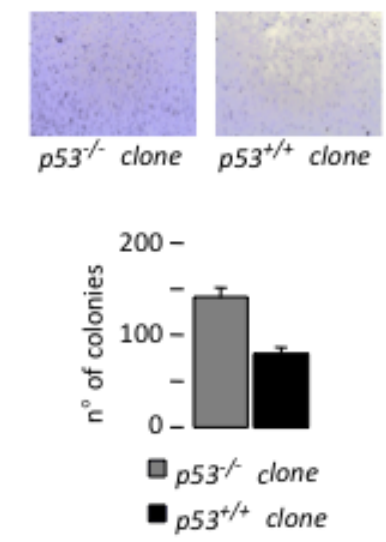

$\mathbf{F}$

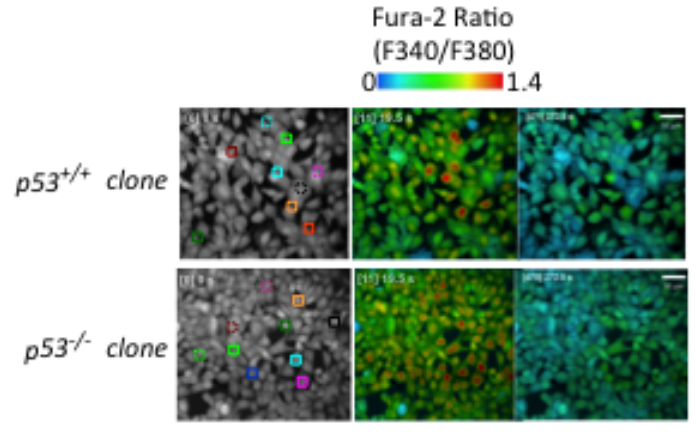

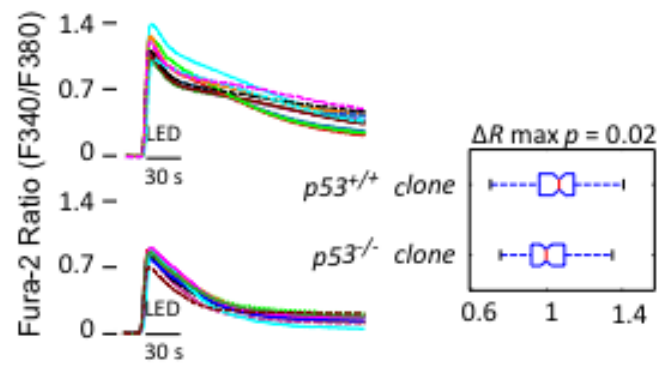

which is a common requirement for tumor suppressors that can regulate ER-to-mitochondria $\mathrm{Ca}^{2+}$ transfer $[6,7,11$ 13].

We generated the following two stable clones to verify the relevance of the modulation of $\mathrm{Ca}^{2+}$ homeostasis by $\mathrm{p} 53$ in the tumor environment: the mouse embryo fibroblast (MEF) $p 53^{-/}$clone that was transduced with $\mathrm{H}-\mathrm{RAS}^{\mathrm{V} 12}$ ( $p 53^{-/}$clone) and the MEF $p 53^{-/-}$clone that was transduced with $\mathrm{H}-\mathrm{RAS}^{\mathrm{V} 12}$ with re-introduced wild type (wt) p53 ( $p 53^{+/+}$clone) (Fig. 1A). We tested the anchorageindependent growth, $\mathrm{Ca}^{2+}$ response and apoptotic sensitivity of these clones in vitro. As expected, the $p 53$ - clone formed approximately twice as many colonies in soft agar (Fig. 1B). Moreover, the $p 53^{-/-}$clone displayed lower mitochondrial $\mathrm{Ca}^{2+}$ uptake after agonist stimulation,

C

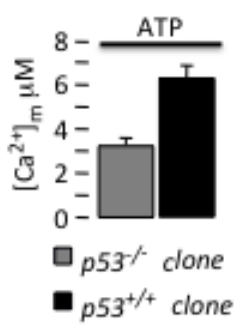

D

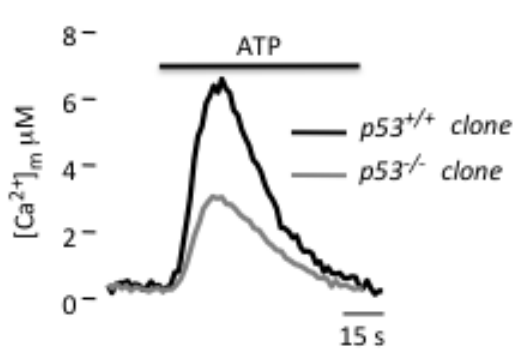

E

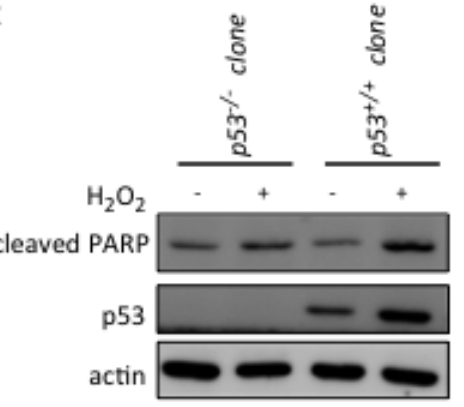

H

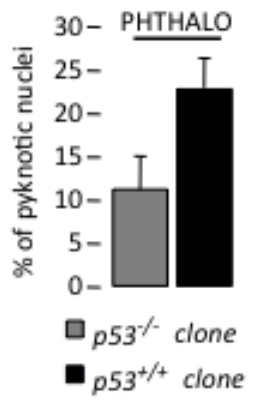

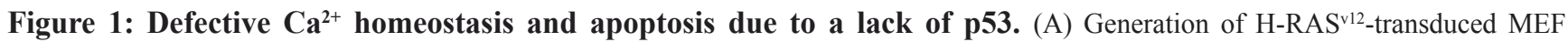
clones. (B) Anchorage-independent growth of H-RAS ${ }^{\mathrm{v} 12}$-transduced MEFs ( $p 53^{+/+}$clone and $p 53^{-/}$clone). Pictures in the upper part are representative of colonies formed. For each well all colonies larger then $0.1 \mathrm{~mm}$ in diameter were counted using ImageJ software. (C) Mitochondrial $\mathrm{Ca}^{2+}$ response obtained with a mitochondria-targeted aequorin chimera after agonist stimulation with ATP $(100 \mu \mathrm{M})\left(p 53^{+/+}\right.$ clone: $\left[\mathrm{Ca}^{2+}\right]_{\mathrm{m}}$ peak $6.23 \pm 0.44 ;$ 53 $^{-/}$clone: $\left[\mathrm{Ca}^{2+}\right]_{\mathrm{m}}$ peak $\left.2.99 \pm 0.21\right)(\mathrm{p}<0.01)$. (D) Representative traces of mitochondrial $\mathrm{Ca}^{2+}$ transient. (E) Apoptotic sensitivity of H-RAS ${ }^{v 12}$-transduced MEF clones $\left(500 \mu \mathrm{M} \mathrm{H}_{2} \mathrm{O}_{2}\right.$ for 6 hours). (F) Cytosolic $\mathrm{Ca}^{2+}$ response of H-RAS ${ }^{\mathrm{v} 12}$ transduced MEF clones loaded with Fura-2 dye upon phthalocyanine $(15 \mu \mathrm{M})$ photo-activation with visible (red) light from a $650 \mathrm{~nm}$ light emitting diode (LED). Microscopic fields of analyzed cells. (G) The ratio of Fura-2 fluorescence $340 \mathrm{~nm} / 380 \mathrm{~nm}$ averaged with the color-matched regions of interest (ROIs) shown in (F), accompanied by statistical analysis. $(\mathrm{H})$ Cell death analysis using the percentage of

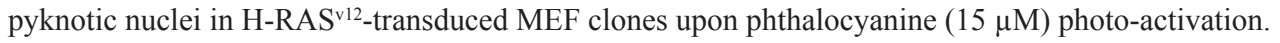


as analyzed by aequorin technology [14], compared to that of the $p 53^{+/+}$clone (Fig. 1C and D). This result indicates that p53 modulates intracellular $\mathrm{Ca}^{2+}$ homeostasis, presumably by blocking the $\mathrm{Ca}^{2+}$ responses generated from the ER, which is a condition that is known to be sufficient to reduce apoptotic sensitivity [15]. Thus, we tested the sensitivity of these two clones to a $\mathrm{Ca}^{2+}$-dependent apoptotic stimulus, $\mathrm{H}_{2} \mathrm{O}_{2}$. As consequence of less $\mathrm{Ca}^{2+}$ mobilization, the $p 53^{-/}$clone exhibited protection from apoptosis when compared to the MEFs clone expressing wt p53 (Fig. 1E). These results suggested that the efficacy of the apoptotic $\mathrm{Ca}^{2+}$ signals is p53-dependent.

The next step was to identify a stimulus that could induce an apoptotic $\mathrm{Ca}^{2+}$ signal within tumor masses using a drug currently applied in clinical cancer therapy. We selected the anti-cancer photosensitizer aluminum phthalocyanine chloride (phthalocyanine) for this purpose. Phthalocyanine is a light-activatable agent used in cancer photodynamic therapy (PDT) [16]. PDT combines a drug called a photosensitizer or photosensitizing agent with a specific type of light to kill cancer cells preferentially because these cells are more prone to accumulate this drug. Indeed, when the photosensitizer drug in tumors absorbs the light, an active form of oxygen is produced that destroys nearby cancer cells [17]. Several photosensitizers have been shown to localize to intracellular organelles, including the ER, where these photosensitizers promote reactive oxygen species (ROS)-mediated cell death upon illumination with suitable wavelengths of visible (red) light [18].

Because different pro-oxidant agents (i.e., $\mathrm{H}_{2} \mathrm{O}_{2}$ and menadione) can produce $\mathrm{Ca}^{2+}$-dependent cell death [5], we hypothesized that phthalocyanine should also produce a $\mathrm{Ca}^{2+}$ signal that results in a toxic effect. Thus, we analyzed the sensitivity of these clones to phthalocyanine in vitro.

Phthalocyanine photo-activation with a LED light resulted in a rapid increase in the cytosolic $\mathrm{Ca}^{2+}$ concentration $\left(\left[\mathrm{Ca}^{2+}\right]_{\mathrm{c}}\right)$ that slowly returned to basal levels, as measured using the ratiometric $\mathrm{Ca}^{2+}$ indicator Fura-2. Interestingly, the $p 53^{--}$clone had an impaired $\mathrm{Ca}^{2+}$ response (Fig. 1F and $\mathrm{G}$, Videos $\mathrm{S} 1$ and $\mathrm{S} 2$ ) and were more resistant in terms of cell death upon phthalocyanine treatment compared to the $p 53^{+/+}$clone, as indicated by the number of pyknotic nuclei appearing after LED irradiation (Fig. 1H).

Next, we tested the involvement of p53 in the

A
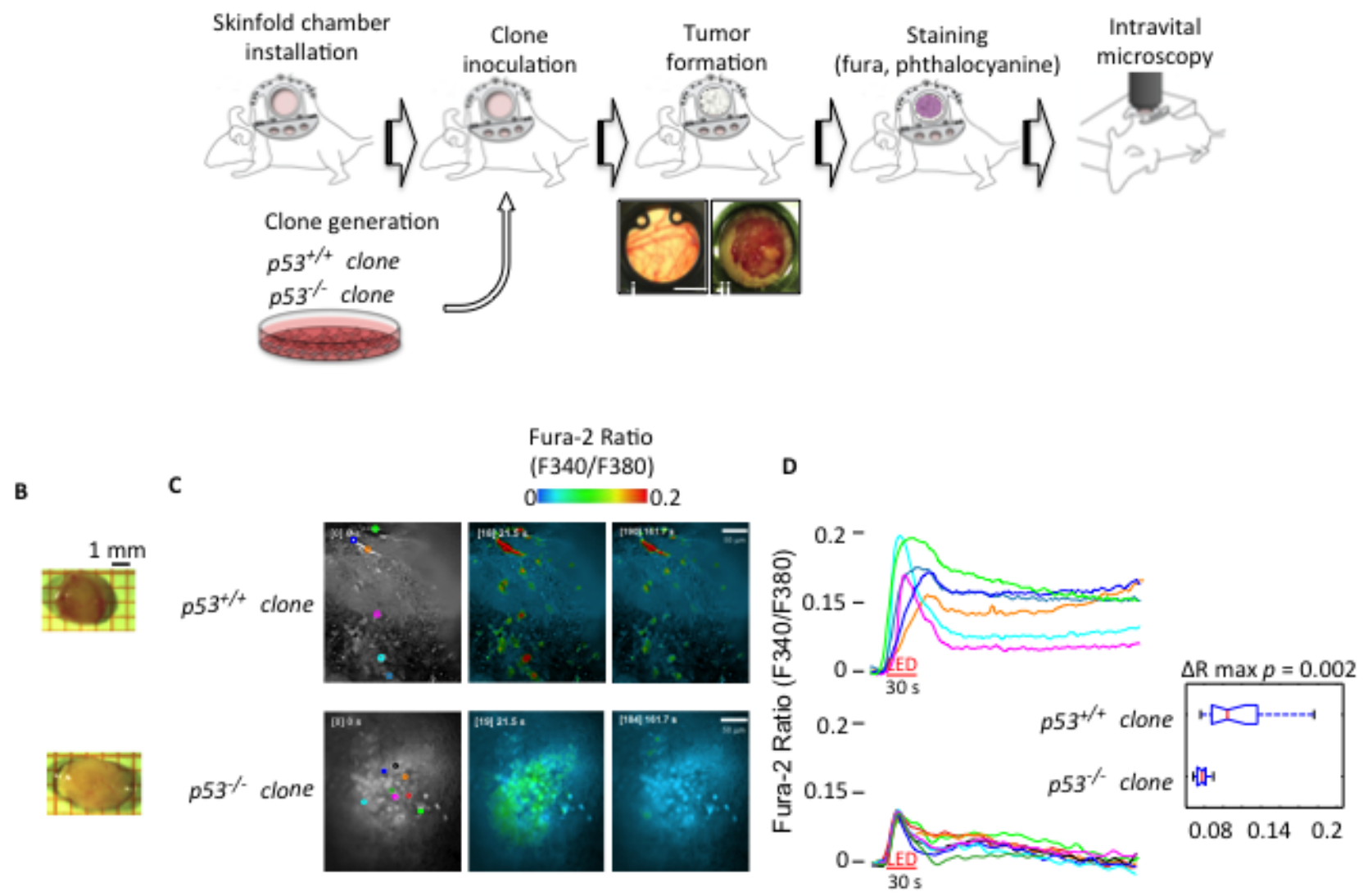

Figure 2: Intravital $\mathrm{Ca}^{2+}$ imaging in tumor masses. (A) Schematic representation of the skinfold chamber technique used to allow tumor formation and subsequent analysis by ratiometric confocal spinning disk intravital microscopy. (B) Representative images of tumor masses originated by the indicated $\mathrm{H}-\mathrm{RAS}^{\mathrm{v} 12}$-transduced MEF clones ( $p 53^{+/+}$and $p 53^{-/}$clones) injected in athymic mice. (C) Cytosolic Ca ${ }^{2+}$ response measured within the tumors obtained by H-RAS ${ }^{\mathrm{v} 12}$-transduced MEF clones injected into skinfold chambers mounted on athymic mice. Microscopic fields of analyzed cells. (D) The ratio of Fura-2 fluorescence $340 \mathrm{~nm} / 380 \mathrm{~nm}$ accompanied by statistical analysis. 
generation of pro-apoptotic $\mathrm{Ca}^{2+}$ signals evoked by cancer PDT in vivo. We developed a new intravital fluorescent microscopy technique to investigate $\mathrm{Ca}^{2+}$ signaling in single cells in tumor masses in live mice during PDT. The MEFs of the two clones were injected either subcutaneously or into a skinfold chamber [19] placed on the dorsal skin of athymic mice [20] (Fig. 2A). Subcutaneous tumors were excised after 2 weeks, and as expected, the $p 53^{--}$clone developed tumors that were double in size compared with the tumors that developed from $p 53^{+/+}$clone (Fig. 2B). Tumors in the dorsal skinfold chamber were loaded with phthalocyanine and the ratiometric $\mathrm{Ca}^{2+}$ indicator Fura-2. $\mathrm{Ca}^{2+}$ signaling was monitored in live mice using a spinning disk confocal microscope (Fig. 2C). Live ratiometric $\mathrm{Ca}^{2+}$ images within the tumor mass and representative traces of the cytosolic $\mathrm{Ca}^{2+}$ response after phthalocyanine photo-activation are shown in Fig. 2C and D and in Videos S3 and S4. Higher $\mathrm{Ca}^{2+}$ responses were evoked in the tumor mass that developed from the $p 53^{+/+}$clone compared to those from the tumor mass that developed from the $p 53^{-/}$clone (Fig. 2C and D, Videos S3 and S4).

These results strongly suggest that the absence of a functional p53 precludes the generation of an efficient $\mathrm{Ca}^{2+}$ response during chemotherapeutic PDT that subsequently prevents the induction of apoptosis to limit tumor growth.

To demonstrate this hypothesis, we modulated the $\mathrm{Ca}^{2+}$ response in vitro and in vivo to confirm the relation between p53-dependent differences in $\mathrm{Ca}^{2+}$ handling and apoptotic behavior. Because we determined that the $p 53^{-/}$MEF clones have a reduced response to PDT due to reduced $\mathrm{Ca}^{2+}$ signaling, we used two different genetic strategies to restore the $\mathrm{Ca}^{2+}$ response and apoptosis: i) overexpression of the mitochondrial $\mathrm{Ca}^{2+}$ uniporter

A
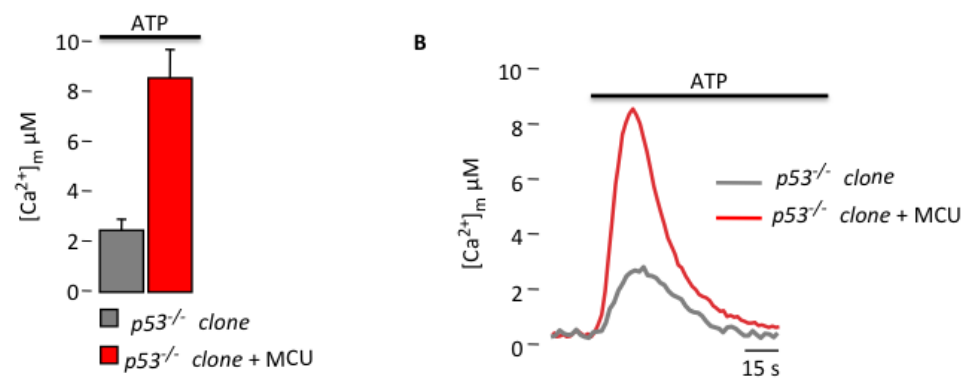

C
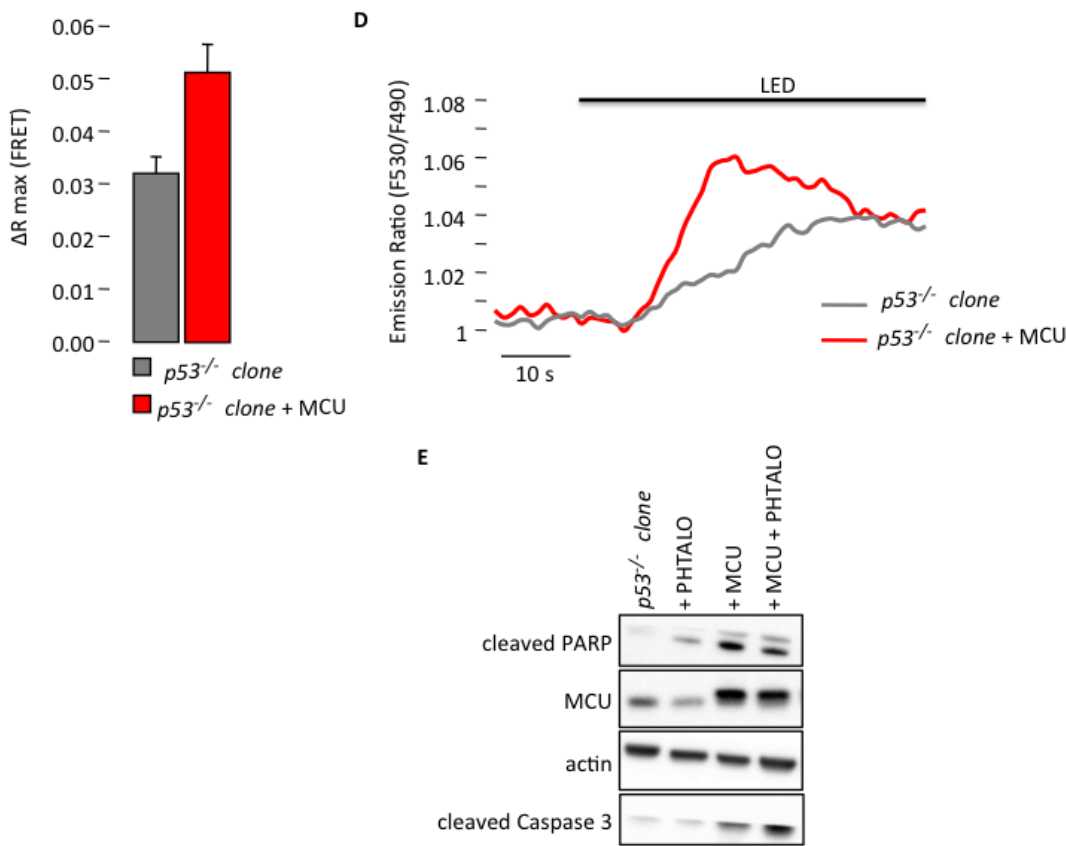

Figure 3: Increased $\mathrm{Ca}^{2+}$ response in $p 53^{-/-} \mathrm{MEF}$ clones after MCU overexpression restores sensitivity to PDT. (A) Mitochondrial $\mathrm{Ca}^{2+}$ response after agonist $(100 \mu \mathrm{M}$ ATP $)$ stimulation of $p 53^{-/}$MEF clone in resting condition or after MCU overexpression ( $553^{--}$clone: $\left[\mathrm{Ca}^{2+}\right]_{\mathrm{m}}$ peak $2.20 \pm 0.45 ;$ 53 $^{-/}$clone $+\mathrm{MCU}$ : $\left[\mathrm{Ca}^{2+}\right]_{\mathrm{m}}$ peak $\left.8.28 \pm 1.45\right)(\mathrm{p}<0.01)$. (B) Representative traces of mitochondrial $\mathrm{Ca}^{2+}$ transient. (C) and (D) Single-cell FRET measurements of PDT-induced mitochondrial $\mathrm{Ca}^{2+}$ uptake that are expressed as the maximal variation in the emission ratio $\left(p 53^{--}\right.$clone: $\Delta \mathrm{R} \max 0.0311 \pm 0.0043 ; p 53^{--}$clone $\left.+\mathrm{MCU}: \Delta \mathrm{R} \max 0.0512 \pm 0.0054\right)(\mathrm{p}<0.05) .(\mathrm{E})$ Immunoblotting for typical apoptotic markers in $p 53^{-/}$clone upon phthalocyanine $(15 \mu \mathrm{M})$ photo-activation (PHTALO). 
(MCU) [21] and ii) overexpression of sarco-ER $\mathrm{Ca}^{2+}$ ATPase pumps (SERCA) [22]. The overexpression of the MCU construct in the $p 53^{-/}$clone increased the ability of mitochondria to accumulate $\mathrm{Ca}^{2+}$. Indeed, the $\left[\mathrm{Ca}^{2+}\right]_{\mathrm{m}}$ rise evoked by agonist stimulation and measured using aequorin technology was increased in the $p 53^{--}$clone overexpressing MCU compared to the $p 53^{--}$clone (Fig. $3 \mathrm{~A}$ and $\mathrm{B})$. These increased values were comparable to those observed in the $p 53^{+/+}$clone (Fig. 1C), which is consistent with previous publications $[23,24]$. We then analyzed the effect of MCU overexpression after PDT. Interestingly, PDT induced a $\mathrm{Ca}^{2+}$ response also in the mitochondrial matrix. In fact, cells transfected with the $\mathrm{Ca}^{2+}$-sensitive FRET-based probe $2 \mathrm{mtD} 3 \mathrm{cpv}$ [25] and treated with phthalocyanine displayed a progressive increase in the FRET ratio (proportional to the mitochondrial $\mathrm{Ca}^{2+}$ concentration) after photo-activationinduced $\mathrm{Ca}^{2+}$ waves. Furthermore, the stimulated $\mathrm{Ca}^{2+}$ uptake by mitochondria was significantly increased after MCU introduction (Fig. 3C and D). This increased
A

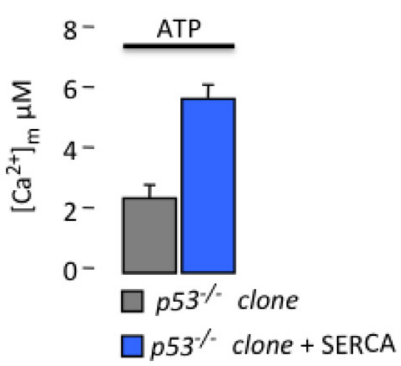

C

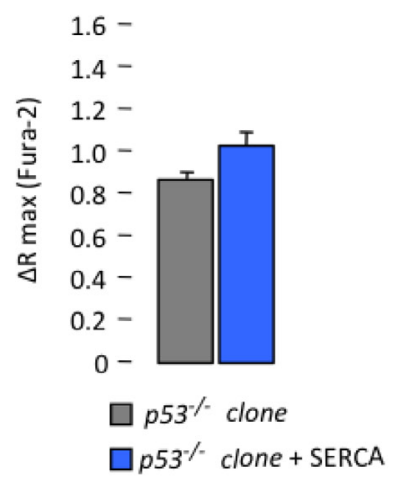

B

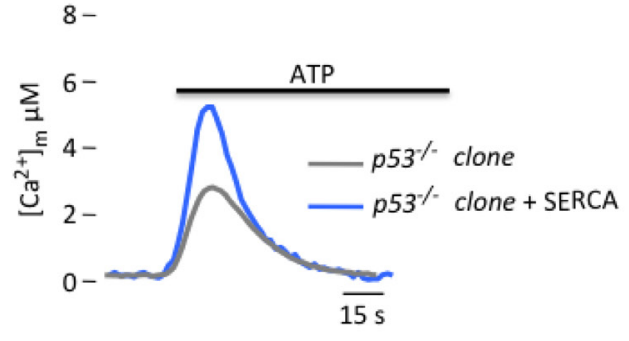

$\mathbf{E}$
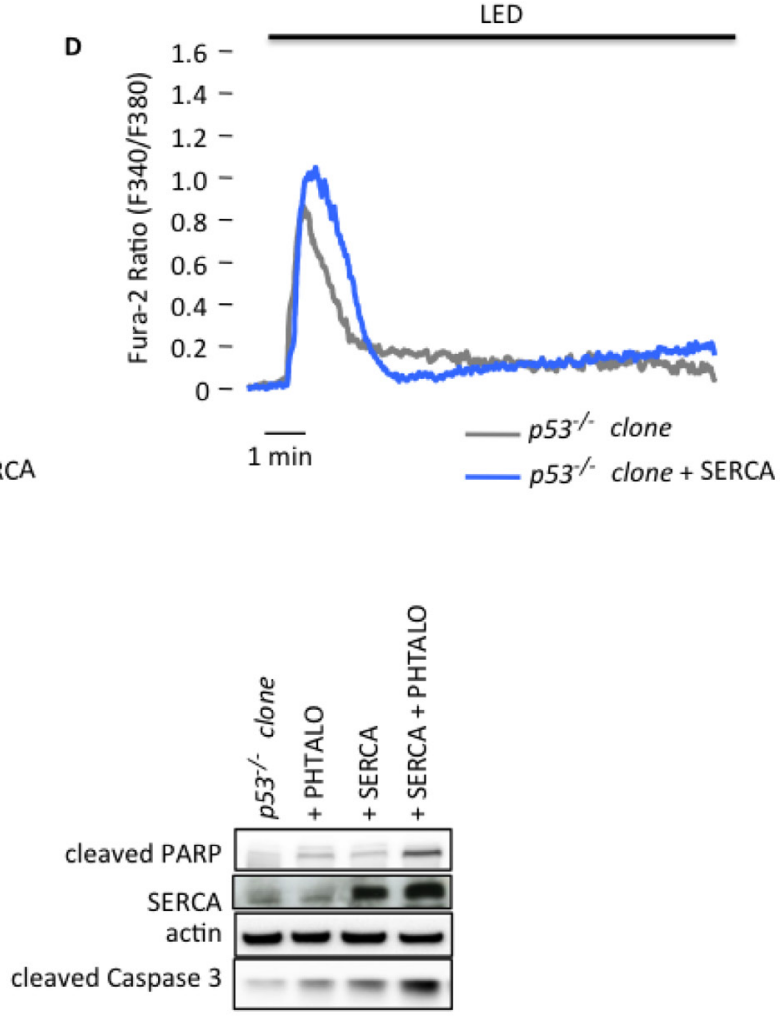

Figure 4: Increased $\mathrm{Ca}^{2+}$ response in $p 53^{-/-}$MEF clones after SERCA overexpression restores sensitivity to PDT. (A) Mitochondrial $\mathrm{Ca}^{2+}$ response after agonist $\left(100 \mu \mathrm{M}\right.$ ATP) stimulation of $p 53^{-/}$clone in resting condition or after SERCA overexpression ( $553^{-}$clone: $\left[\mathrm{Ca}^{2+}\right]_{\mathrm{m}}$ peak $2.51 \pm 0.31 ; p 53^{-}$clone + SERCA: $\left[\mathrm{Ca}^{2+}\right]_{\mathrm{m}}$ peak $\left.5.40 \pm 0.56\right)(\mathrm{p}<0.01)$. (B) Representative traces of mitochondrial $\mathrm{Ca}^{2+}$ transient. (C) and (D) Ratiometric single-cell measurements of PDT-induced $\mathrm{Ca}^{2+}$ waves by Fura-2 that are expressed as the maximal variation in the excitation ratio ( $p 53^{-/}$clone: $\Delta \mathrm{R} \max 0.85 \pm 0.02 ; p 53^{-/}$clone + SERCA: $\left.\Delta \mathrm{R} \max 1.05 \pm 0.06\right)(\mathrm{p}<0.01)$. (E) Immunoblotting for typical apoptotic markers in $p 53^{-/}$clone upon phthalocyanine $(15 \mu \mathrm{M})$ photo-activation (PHTALO). 
A

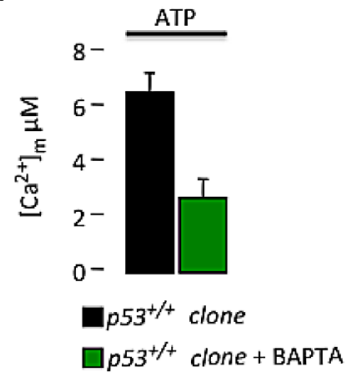

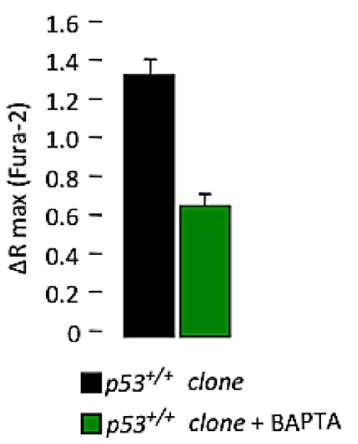

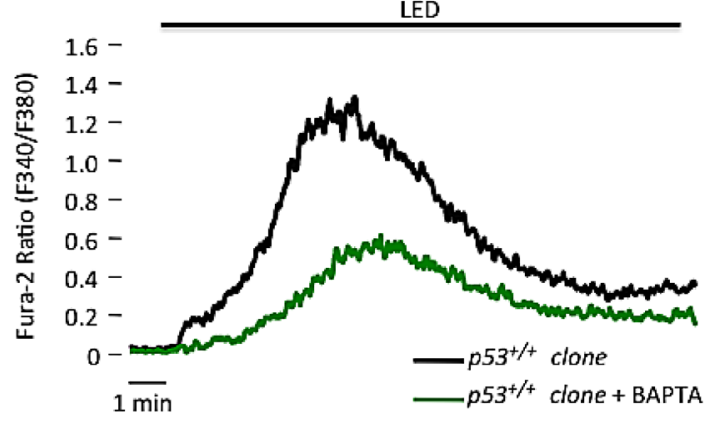

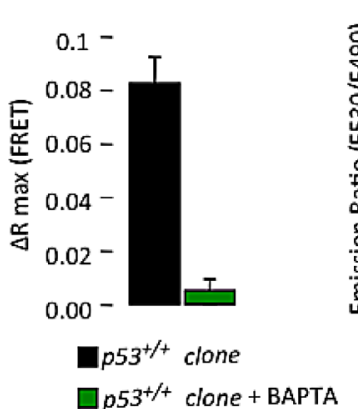

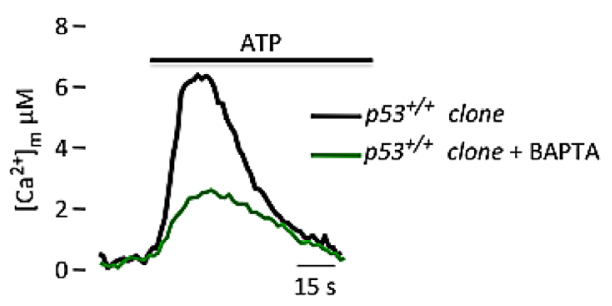


mitochondrial $\mathrm{Ca}^{2+}$ responsiveness was associated with a re-established apoptotic sensitivity induced by PDT in vitro (Fig. $3 \mathrm{E}$ ). As additional approach we used the overexpression of SERCA. Indeed its increased activity, and the subsequent ER $\mathrm{Ca}^{2+}$ overload have been previously demonstrated to promote apoptosis $[5,26]$. In our studies, the overexpression of SERCA pumps in the $p 53^{-/}$clone restored the mitochondrial $\mathrm{Ca}^{2+}$ response after agonist stimulation (Fig. 4A and B) as in the $p 53^{+/+}$clone (Fig. 1C). Similar to the results obtained after MCU overexpression, also PDT-evoked $\mathrm{Ca}^{2+}$ release from the ER into the cytosol was increased (Fig. 4C and D), as measured using the ratiometric $\mathrm{Ca}^{2+}$ indicator Fura-2. Consequently, apoptotic sensitivity was rescued in the cells overexpressing SERCA pumps (Fig. 4E). These results support the evidence that the alteration of $\mathrm{Ca}^{2+}$ homeostasis observed in the absence of $\mathrm{p} 53$, is determinant for sensitivity to apoptosis and that modulating mitochondrial $\mathrm{Ca}^{2+}$ handling is possible to resensitize cells to death.

Moreover, we used an approach for preventing $\mathrm{Ca}^{2+}$ signaling [27] in the $p 53^{+/+}$clone to mimic the $\mathrm{Ca}^{2+}$ signaling conditions observed both in vitro and in vivo in the $p 53^{--}$clone to test the dependency of PDT-induced apoptosis on mitochondrial $\mathrm{Ca}^{2+}$ uptake. Using BAPTA-
A
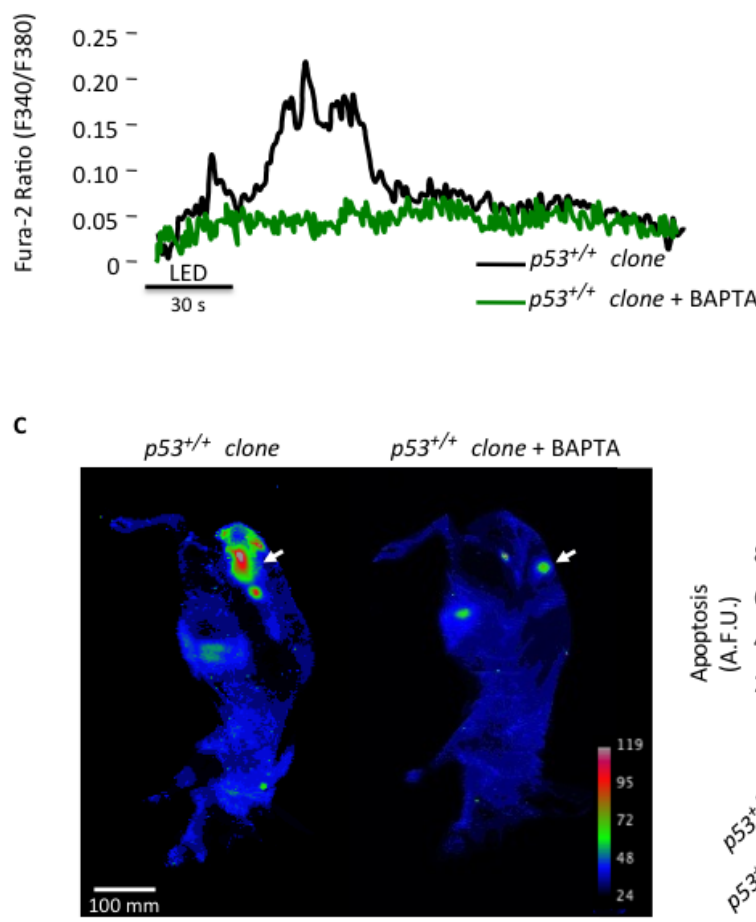

B

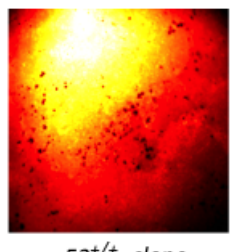

$p 53^{+/+}$clone

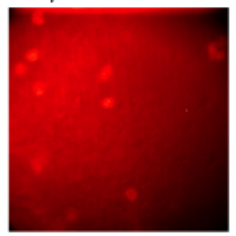

$p 53^{+/+}$clone + BAPTA

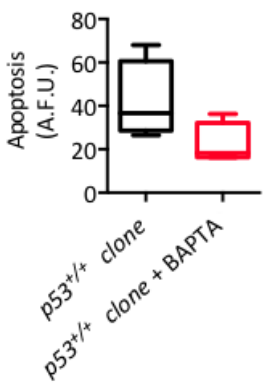

D
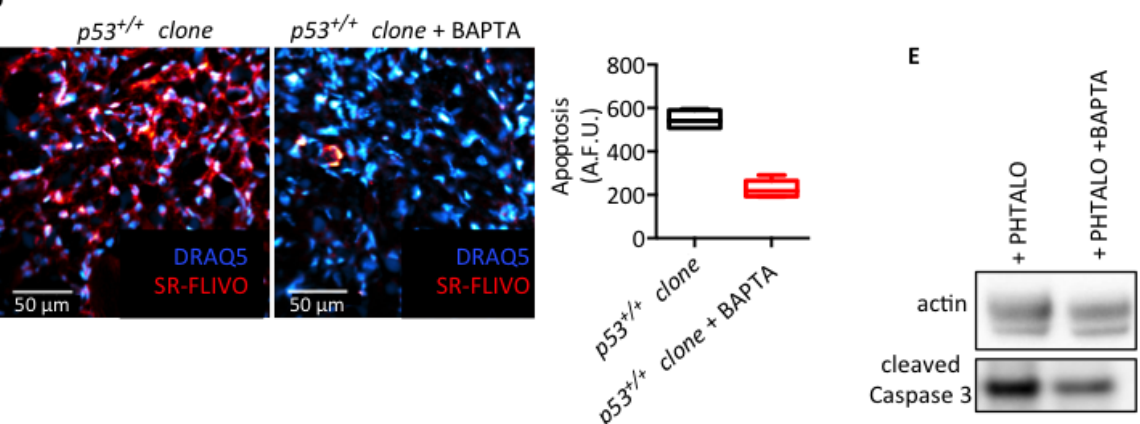

Figure 6: $\mathrm{Ca}^{2+}$ signal in tumor masses is required for the anticancer effect of PDT. (A) Cytosolic Ca ${ }^{2+}$ response measured within the tumors in the skinfold chambers as the ratio of Fura- 2 fluorescence $340 \mathrm{~nm} / 380 \mathrm{~nm}$ induced upon phthalocyanine (15 $\mu \mathrm{M}$ ) photoactivation. (B) Levels of apoptosis measured as caspase activity (SR-FLIVO) within the tumor masses in the skinfold chambers. (C) In vivo imaging of apoptosis as the intensity of fluorescence (SR-FLIVO) emitted by a subcutaneous tumor mass upon phthalocyanine (15 $\mu \mathrm{M})$ photo-activation. (D) Analysis of apoptosis in tumor tissue sections prepared upon in vivo phthalocyanine (15 $\mu \mathrm{M})$ photo-activation and tumor excision. (E) Immunoblotting of homogenized tumors excised upon in vivo phthalocyanine $(15 \mu \mathrm{M})$ photo-activation. 
AM, which is a well-known membrane permeable $\mathrm{Ca}^{2+}$ chelator, we were able to reduce the $\left[\mathrm{Ca}^{2+}\right]_{\mathrm{m}}$ response evoked by agonist stimulation (Fig. $5 \mathrm{~A}$ and $\mathrm{B}$ ) to values similar to those observed in the $p 53^{-/}$clone (Fig. 1C). We further assessed the effect of the chelator after PDT treatment on either the cytosolic (Fig. 5C and D) or mitochondrial $\mathrm{Ca}^{2+}$ response (Fig. 5E and $\mathrm{F}$ ). The reduced ability of mitochondria to accumulate $\mathrm{Ca}^{2+}$ was associated with reduced sensitivity to PDT-induced apoptosis (Fig. $5 \mathrm{G}$ ) as observed previously in the $p 53^{-/}$clone (Fig. $1 \mathrm{H}$ ), probably due to a defective opening of the mitochondrial permeability transition pore $[27,28]$.

Then, we also validated our findings in vivo. First, we directly verified in the tumor masses (derived from the $p 53^{+/+}$clone) grown in skinfold chambers and found that the activation of phthalocyanine was unable to induce a significant increase in $\mathrm{Ca}^{2+}$ concentration in the presence of BAPTA (Fig. 6A). Then, we directly linked $\mathrm{Ca}^{2+}$ response with apoptotic behavior using SR-FLIVO, which is a fluorescent probe used to detect apoptosis in vivo. Indeed, as shown in the lower panel of Fig. 6B, tumors treated with PDT exhibited reduced levels of caspase activity in the presence of BAPTA compared to levels of apoptosis in tumors not exposed to this $\mathrm{Ca}^{2+}$ chelator (Fig. 6B, upper panel). To further investigate the correlation between $\mathrm{Ca}^{2+}$ and apoptosis in vivo, two groups of mice were injected subcutaneously with the $p 53^{+/+}$clones to induce tumor formation. After 14 days, the animals were pre-treated with a control (vehicle) or with BAPTA-AM for 1 hour at the level of the tumor nodules, irradiated with PDT and then injected with SR-FLIVO to image caspase-positive tumor cells. Fig. 6C clearly shows that the inhibition of $\mathrm{Ca}^{2+}$ signals reduced apoptotic activation in vivo (as previously observed in vitro). Finally, tumor nodules were excised and imaged by confocal microscopy (Fig. 6D) or homogenized for Western blot analysis (Fig. 6E). Tumor cells treated with BAPTA displayed significantly decreased fluorescence (and thus, reduced apoptosis due to the inhibition of caspase activation) compared to that of the tumors not treated with BAPTA $\left(p 53^{+/+}\right.$clone). These results were confirmed by immunoblotting the extracted tumors with anti-caspase 3 antibody (Fig. 6E).

Taken together, these results indicated that the deregulation of $\mathrm{Ca}^{2+}$ signaling in the absence of $\mathrm{p} 53$ reduces the sensitivity of tumor cells to PDT-induced apoptosis.

\section{DISCUSSION}

Our ability to elucidate the details of intracellular signaling has improved remarkably in recent years. Technological innovations resulting from the development of new specialized optical microscopes and from the introduction of fluorescent indicators and proteins allow us to visualize events within a cell in real time and space [14]. These tools provide high sensitivity and great versatility while minimally perturbing the cell under investigation.
Much of the recent progress in understanding the biology of cancer cells has been achieved via the study of key components of signaling and regulatory pathways. A widely studied signal transduction pathway in cancer cells is intracellular $\mathrm{Ca}^{2+}$ homeostasis [15]. $\mathrm{Ca}^{2+}$ plays roles in both tumor progression and the efficacy of chemotherapeutic agents [1], and the participation of this cation in the apoptotic process has been extensively demonstrated [16]. The link of $\mathrm{Ca}^{2+}$ to cancer and apoptosis was initially accepted with the discovery that the classical antiapoptotic protein $\mathrm{Bcl}-2$ affected $\mathrm{Ca}^{2+}$ signaling. In particular, the antiapoptotic members of the Bcl-2 family cause a reduction in the ER $\mathrm{Ca}^{2+}$ concentration, while the opposite is true for proapoptotic proteins. Indeed, apoptotic sensitivity correlates with the total ER $\mathrm{Ca}^{2+}$ load and depends on the ability of cells to transfer $\mathrm{Ca}^{2+}$ from the ER to the cytosol [17]. Strikingly, proteins other than the proapoptotic members of the Bcl2 family act by modulating $\mathrm{Ca}^{2+}$ homeostasis. Indeed, in recent years, many other onco-suppressor proteins have been shown to reduce the $\mathrm{Ca}^{2+}$ release in the ER (albeit through completely different molecular mechanisms) and, consequently, to block the apoptotic responses [5,6,18-20]. However, all of these mechanisms have been successfully elucidated in vitro. In contrast, no data regarding the role of intracellular $\mathrm{Ca}^{2+}$ homeostasis are available in the context of the tumor environment in vivo in mice.

In the present study, we combined the dorsal skinfold chamber technique with intravital microscopy to elucidate the involvement of p53 in the control of intracellular $\mathrm{Ca}^{2+}$ signals and apoptosis in three-dimensional tumor masses in living mice. The obtained data strongly indicate that p53-dependent dysregulated $\mathrm{Ca}^{2+}$ homeostasis causes reduced $\mathrm{ER} \mathrm{Ca}^{2+}$ release that is associated with reduced responsivity to apoptotic stimulation. More interestingly, we showed that $\mathrm{Ca}^{2+}$ signals with amplitudes that are directly dependent on the presence of p53 and that are tightly linked with the generation of apoptosis in cancer cells are generated when the anti-cancer photosensitizer phthalocyanine, which is used in the photodynamic therapy of cancer, is activated.

To our knowledge, this study is the first to report the direct measurements of intracellular $\mathrm{Ca}^{2+}$ dynamics in vivo within tumor masses under physiological conditions. Moreover, this imaging approach can be applied successfully to all the various GFP moieties and chimeras, as well as to all of the fluorescent probes that are available, to follow intracellular parameters of interest.

Overall, these results provide new insights into p53 pathways underlying cancer and a method to monitor the kinetics of intracellular signals in tumor masses in vivo to verify the efficacy of anticancer treatments. 


\section{MATERIALS AND METHODS}

\section{Reagents and solutions}

ATP, histamine, digitonin, $\mathrm{H}_{2} \mathrm{O}_{2}$, and phthalocyanine were purchased from Sigma-Aldrich. Coelenterazine was purchased from Synchem. Fura-2 AM and BAPTA-AM were purchased from Invitrogen.

Krebs-Ringer bicarbonate solution (KRB) contained $125 \mathrm{mM} \mathrm{NaCl}, 5 \mathrm{mM} \mathrm{KCl}, 1 \mathrm{mM} \mathrm{MgSO}_{4}, 1$ $\mathrm{mM} \mathrm{Na} \mathrm{HPO}_{4}, 5.5 \mathrm{mM}$ glucose, $20 \mathrm{mM} \mathrm{NaHCO}, 2$ $\mathrm{mM}$ l-glutamine and $20 \mathrm{mM}$ HEPES (pH 7.4) and was supplemented with $1 \mathrm{mM} \mathrm{CaCl}_{2}$.

\section{Cell culture, transfection and cell death induction}

HeLa cells were obtained from the American Type Culture Collection. The HeLa cells were cultured in Dulbecco's modified Eagle's medium (DMEM) supplemented with $10 \%$ FCS and transfected using a standard calcium phosphate procedure.

The MEFs were generated by crossing $p 53^{+/}$mice and collecting cells from 13.5 d.p.c. embryos. The MEFs were cultured in DMEM supplemented with $10 \%$ FCS and treated with $500 \mu \mathrm{M} \mathrm{H}_{2} \mathrm{O}_{2}$ for 6 hours or with $15 \mu \mathrm{M}$ phthalocyanine for 2 hours in complete medium.

\section{Detection of cell death}

In vitro. Apoptosis was determined by different methods as indicated in the text: (i) by blotting for cell death markers, such as cleaved PARP or cleaved Caspase 3 , or (ii) by the automated detection of pyknotic nuclei. Briefly, cells were fixed and stained with DAPI, and images were acquired using an automated microscope as previously described. Then, pyknotic nuclei were identified by combining DAPI intensity and the nucleus form factor.

In vivo. After an i.v. injection of $100 \mu \mathrm{l}$ of SRFLIVO $^{\text {TM }}$ (Immunochemistry Technologies) via the lateral tail vein, the FLIVO reagent was allowed to circulate in the mouse for 30 minutes before analysis. Fluorescent in vivo images were acquired using an Odessey ${ }^{\circledR}$ CLx Imaging System (Li-Cor). Then, the tumors were excised, frozen, sectioned and stained for nuclei using DRAQ5 according to the manufacturer's protocol (Cell Signaling). After staining, the samples were mounted on coverslips and analyzed using a Zeiss LSM 510 confocal microscope equipped with a Fluor 40x/1.30 oil immersion objective. The acquired images were background corrected, and signals were analyzed using the open source Fiji software (available at http://fiji.sc/Fiji).

\section{Aequorin measurements}

Cells grown on $13 \mathrm{~mm}$ round glass coverslips at $50 \%$ confluence were transfected with the appropriate chimera cytAEQ or mtAEQ as previously described [7] alone or together with p53 wt expression constructs.

All aequorin measurements were performed in KRB. Agonists were added to the same medium as specified in the figure legends. The experiments were terminated by lysing the cells with $100 \mu \mathrm{M}$ digitonin in a hypotonic $\mathrm{Ca}^{2+}$ rich solution $\left(10 \mathrm{mM} \mathrm{CaCl}_{2}\right.$ in $\left.\mathrm{H}_{2} \mathrm{O}\right)$, thus discharging the remaining aequorin pool. The light signals were collected and calibrated to $\left[\mathrm{Ca}^{2+}\right]$ values as previously described [7].

\section{Fura-2 measurements}

The cytosolic $\mathrm{Ca}^{2+}$ response was evaluated using the fluorescent $\mathrm{Ca}^{2+}$ indicator Fura-2/AM (Life Technologies, Invitrogen). The MEFs were grown on 24-mm coverslips and incubated at $37^{\circ} \mathrm{C}$ for 30 minutes in $1 \mathrm{mM} \mathrm{Ca}^{2+} / \mathrm{KRB}$ supplemented with $2.5 \mathrm{mM}$ Fura-2/AM, 0.02\% Pluronic F-68 (Sigma-Aldrich), and $0.1 \mathrm{mM}$ sulfinpyrazone (Sigma-Aldrich). Then, the cells were washed and supplied with $1 \mathrm{mM} \mathrm{Ca}^{2+} / \mathrm{KRB}$. Next, the cells were placed in an open Leyden chamber on a $37{ }^{\circ} \mathrm{C}$ thermostated stage and exposed to $340 / 380$ wavelength light using the Olympus xcellence (Olympus) multiple wavelength highresolution fluorescence microscopy system equipped with an Hamamatsu ORCA ER CCD camera (Hamamatsu Photonics) and a Upl FLN 40x oil objective (Olympus) to determine the cytosolic $\mathrm{Ca}^{2+}$ response. The photoactivation of aluminum phthalocyanine chloride was obtained using an excitation filter ET576/25 (Semrock), with 500 milliseconds of excitation every FRET ratio cycle. The collected fluorescence data are expressed as emission ratios.

\section{FRET-based mitochondrial $\mathrm{Ca}^{2+}$ measurements}

MEF cells were grown on 24-mm coverslips transfected with $4 \mathrm{mtD} 3 \mathrm{cpv}$. After 36 hours, the cells were imaged using a Zeiss Axiovert 200M microscope with a cooled CCD camera (Photometrics), which was equipped with a C-apochromatic 40x/1.2 W CORR objective and controlled by MetaFluor 7.0 software (Universal Imaging). Emission ratio imaging of $4 \mathrm{mtD} 3 \mathrm{cpv}$ was accomplished using a 436DF20 excitation filter, a 450 $\mathrm{nm}$ dichroic mirror, and two emission filters (475/40 for ECFP and 535/25 for citrine) that were controlled by a Lambda 10-2 filter changer (Sutter Instruments, Novato, CA 94949, USA). The acquired fluorescence images were background corrected. The exposure times were typically 100-200 milliseconds, and images were collected every second per wavelength. The photo-activation of aluminum 
phthalocyanine chloride was achieved using an excitation filter ET650/50 (Chroma Technology Corp.), with 500 milliseconds of excitation every FRET ratio cycle.

\section{Transformation assays and in vivo tumorigenicity}

Low-passage MEF clones infected with H-RAS ${ }^{\mathrm{V} 12}$ or $\mathrm{H}-\mathrm{Ras}^{\mathrm{V} 12}+\mathrm{p} 53$ wt were resuspended in DMEM that was supplemented with $10 \%$ fetal bovine serum, penicillin $(100 \mathrm{IU} / \mathrm{ml})$ and streptomycin $(100 \mathrm{IU} / \mathrm{ml})$ and that contained $0.3 \%$ agarose. Then, the cells were plated on top of $1 \%$ agarose in the same medium at a cell density of $1 \times 10^{5}$ for each $60 \mathrm{~mm}$ plate. Colonies reaching at least $100 \mu \mathrm{m}$ were scored after 14 days in culture. These experiments were performed in triplicate.

To assess the tumorigenicity of $\mathrm{H}-\mathrm{RAS}^{\mathrm{V} 12}$-infected MEFs, 1 X $10^{6}$ cells were injected subcutaneously on both sides of athymic mice, and tumors were excised after 2 weeks. Procedures involving animals and their care conformed to institutional guidelines, and all experimental protocols were approved by the Animal Ethics Committee.

\section{Dorsal skinfold chamber and intravital microscopy}

Dorsal skin-fold chambers were transplanted onto 7-week-old female athymic mice (Harlan), as described [8]. A cell pellet containing $10^{6}$ tumor cells was placed onto the tissue surface (drop-on method) at three days postsurgery. Intravital microscopy analyses were performed two weeks after cell inoculation as follows: the mice were anesthetized by halothane and transferred to a custommade warmed plate mounted on the stage of an upright spinning disk confocal system microscope (Olympus DSU) equipped with a water immersion objective (Fluor 40x, 1.0 N.A., Nikon) for observation. The AM ester of Fura-2 $(15 \mu \mathrm{M})$ and aluminum phthalocyanine chloride (dissolved in DMS at a final concentration of $15 \mu \mathrm{M}$ ) were loaded into each tumor mass by pressure injection with a glass microcapillary formed on a pipette puller (PP-830, Narishige). Cytosolic $\mathrm{Ca}^{2+}$ signals were tracked in the dorsal skinfold chamber by the sequential illumination of Fura-2 at $340 \mathrm{~nm}$ and $380 \mathrm{~nm}$ from two light emitting diodes. Fluorescence images were formed on the scientific complementary metal-oxide-semiconductor (sCMOS) sensor of a high-performance camera (pco.edge) under the control of software written in the laboratory. Images were acquired in vivo before, during and after the photoactivation of aluminum phthalocyanine chloride with light from a $650 \mathrm{~nm}$ light emitting diode placed immediately above the microscope objective and directed onto the sample by reflection off a dichromatic short-pass mirror (Edmund Optics, part n. 69-217). The acquired images were processed off-line by software written in the laboratory using the Matlab environment (Release
14, The MathWorks, Inc., Natick, MA, USA). Signals were measured as dye emission ratio changes using the following equation: $\Delta R=R(\mathrm{t})-R(0)$, where $t$ is time, $R(t)$ is the emission intensity excited at $340 \mathrm{~nm}$ divided by the intensity excited at $380 \mathrm{~nm}$, and $R(0)$ indicates the prestimulus ratio.

For the BAPTA-AM experiments, $1 \mu \mathrm{M}$ BAPTA was loaded together with Fura-2 and phthalocyanine into each tumor mass. For the in vivo detection of apoptosis in the dorsal skinfold chamber, SR-FLIVO (see previous paragraph) was injected in situ and incubated for 60 minutes in the exposed area, and excess fluorophore was extensively washed with PBS. Then, images were acquired using the previously described imaging system, which was equipped with a Zeiss EC Plan-Neo fluor 5x/0.16 objective.

\section{Statistical analysis of data}

The statistical data are presented as the mean \pm SE. Significance was calculated by Student's t-test, and correlation analysis was conducted using SigmaPlot 5.0 software (SPSS Inc.).

\section{ACKNOWLEDGMENTS AND GRANT SUPPORT}

This study was supported by the Italian Association for Cancer Research (AIRC), by local funds from the University of Ferrara to P.P. and C.G., by the Italian Ministry of Education, University and Research (COFIN, FIRB, and Futuro in Ricerca) to P.P., and by NCI grants to P.P. We thank Paola Braghetta, Alessandra Ferlini and Catalin Ciubotaru for their help in running some experiments.

\section{Conflict of interest}

The authors declare no conflicts of interest related to this work.

\section{REFERENCES}

1. Miliani de Marval PL and Zhang Y. The RP-Mdm2-p53 pathway and tumorigenesis. Oncotarget. 2011; 2(3):234238.

2. Orrenius S, Zhivotovsky B and Nicotera P. Regulation of cell death: the calcium-apoptosis link. NatRevMolCell Biol. 2003; 4(7):552-565.

3. Giorgi C, Baldassari F, Bononi A, Bonora M, De Marchi E, Marchi S, Missiroli S, Patergnani S, Rimessi A, Suski JM, Wieckowski MR and Pinton P. Mitochondrial $\mathrm{Ca}(2+)$ and apoptosis. Cell Calcium. 2012; 52(1):36-43.

4. Rudolf R, Mongillo M, Magalhaes PJ and Pozzan T. In vivo 
monitoring of $\mathrm{Ca}(2+)$ uptake into mitochondria of mouse skeletal muscle during contraction. J Cell Biol. 2004; 166(4):527-536.

5. Pinton P, Ferrari D, Rapizzi E, Di Virgilio FD, Pozzan T and Rizzuto $\mathrm{R}$. The $\mathrm{Ca} 2+$ concentration of the endoplasmic reticulum is a key determinant of ceramide-induced apoptosis: significance for the molecular mechanism of Bcl2 action. EMBO J. 2001; 20(11):2690-2701.

6. Giorgi C, Ito K, Lin HK, Santangelo C, Wieckowski MR, Lebiedzinska M, Bononi A, Bonora M, Duszynski J, Bernardi R, Rizzuto R, Tacchetti C, Pinton P and Pandolfi PP. PML regulates apoptosis at endoplasmic reticulum by modulating calcium release. Science. 2010; 330(6008):1247-1251.

7. Bononi A, Bonora M, Marchi S, Missiroli S, Poletti F, Giorgi C, Pandolfi PP and Pinton P. Identification of PTEN at the ER and MAMs and its regulation of Ca signaling and apoptosis in a protein phosphatase-dependent manner. Cell Death Differ. 2013.

8. Zilfou JT and Lowe SW. Tumor suppressive functions of p53. Cold Spring Harbor perspectives in biology. 2009; 1(5):a001883.

9. Marouco D, Garabadgiu AV, Melino G and Barlev NA. Lysine-specific modifications of p53: a matter of life and death? Oncotarget. 2013; 4(10):1556-1571.

10. Sorrentino G, Mioni M, Giorgi C, Ruggeri N, Pinton P, Moll U, Mantovani F and Del Sal G. The prolyl-isomerase Pin1 activates the mitochondrial death program of p53. Cell Death Differ. 2013; 20(2):198-208.

11. Scorrano L, Oakes SA, Opferman JT, Cheng EH, Sorcinelli $\mathrm{MD}$, Pozzan $\mathrm{T}$ and Korsmeyer SJ. BAX and BAK regulation of endoplasmic reticulum $\mathrm{Ca} 2+$ : a control point for apoptosis. Science. 2003; 300(5616):135-139.

12. Echeverry N, Bachmann D, Ke F, Strasser A, Simon HU and Kaufmann T. Intracellular localization of the BCL2 family member BOK and functional implications. Cell Death Differ. 2013; 20(6):785-799.

13. Rimessi A, Marchi S, Fotino C, Romagnoli A, Huebner $\mathrm{K}$, Croce CM, Pinton P and Rizzuto R. Intramitochondrial calcium regulation by the FHIT gene product sensitizes to apoptosis. Proc Natl Acad Sci U S A. 2009; 106(31):1275312758.

14. Bonora M, Giorgi C, Bononi A, Marchi S, Patergnani S, Rimessi A, Rizzuto R and Pinton P. Subcellular calcium measurements in mammalian cells using jellyfish photoprotein aequorin-based probes. Nat Protoc. 2013; 8(11):2105-2118.

15. Pinton P, Giorgi C, Siviero R, Zecchini E and Rizzuto R. Calcium and apoptosis: ER-mitochondria $\mathrm{Ca} 2+$ transfer in the control of apoptosis. Oncogene. 2008; 27(50):64076418 .

16. Agostinis P, Berg K, Cengel KA, Foster TH, Girotti AW, Gollnick SO, Hahn SM, Hamblin MR, Juzeniene A, Kessel D, Korbelik M, Moan J, Mroz P, Nowis D, Piette J, Wilson
BC, et al. Photodynamic therapy of cancer: an update. CA: a cancer journal for clinicians. 2011; 61(4):250-281.

17. Dolmans DE, Fukumura D and Jain RK. Photodynamic therapy for cancer. Nat Rev Cancer. 2003; 3(5):380-387.

18. Shahzidi S, Cunderlikova B, Wiedlocha A, Zhen Y, Vasovic V, Nesland JM and Peng Q. Simultaneously targeting mitochondria and endoplasmic reticulum by photodynamic therapy induces apoptosis in human lymphoma cells. Photochemical \& photobiological sciences : Official journal of the European Photochemistry Association and the European Society for Photobiology. 2011; 10(11):17731782.

19. Sckell A and Leunig M. The dorsal skinfold chamber: studying angiogenesis by intravital microscopy. Methods Mol Biol. 2009; 467:305-317.

20. Lehr HA, Leunig M, Menger MD, Nolte D and Messmer K. Dorsal skinfold chamber technique for intravital microscopy in nude mice. Am J Pathol. 1993; 143(4):1055-1062.

21. Marchi S and Pinton $\mathrm{P}$. The mitochondrial calcium uniporter complex: molecular components, structure and physiopathological implications. The Journal of physiology. 2014; 592(Pt 5):829-839.

22. Bublitz M, Musgaard M, Poulsen H, Thogersen L, Olesen C, Schiott B, Morth JP, Moller JV and Nissen P. Ion pathways in the sarcoplasmic reticulum $\mathrm{Ca} 2+$-ATPase. J Biol Chem. 2013; 288(15):10759-10765.

23. De Stefani D, Raffaello A, Teardo E, Szabo I and Rizzuto R. A forty-kilodalton protein of the inner membrane is the mitochondrial calcium uniporter. Nature. 2011.

24. Baughman JM, Perocchi F, Girgis HS, Plovanich M, Belcher-Timme CA, Sancak Y, Bao XR, Strittmatter L, Goldberger O, Bogorad RL, Koteliansky V and Mootha VK. Integrative genomics identifies MCU as an essential component of the mitochondrial calcium uniporter. Nature. 2011.

25. Palmer AE, Giacomello M, Kortemme T, Hires SA, LevRam V, Baker D and Tsien RY. Ca2+ indicators based on computationally redesigned calmodulin-peptide pairs. Chemistry \& biology. 2006; 13(5):521-530.

26. Brini M, Bano D, Manni S, Rizzuto R and Carafoli E. Effects of PMCA and SERCA pump overexpression on the kinetics of cell $\mathrm{Ca}(2+)$ signalling. EMBO J. 2000; 19(18):4926-4935.

27. Hajnoczky G, Csordas G, Das S, Garcia-Perez C, Saotome M, Sinha Roy S and Yi M. Mitochondrial calcium signalling and cell death: approaches for assessing the role of mitochondrial $\mathrm{Ca} 2+$ uptake in apoptosis. Cell Calcium. 2006; 40(5-6):553-560.

28. Bonora M, Bononi A, De Marchi E, Giorgi C, Lebiedzinska M, Marchi S, Patergnani S, Rimessi A, Suski JM, Wojtala A, Wieckowski MR, Kroemer G, Galluzzi L and Pinton P. Role of the c subunit of the FO ATP synthase in mitochondrial permeability transition. Cell cycle. 2013; 12(4):674-683. 